\title{
Atividade elétrica dos músculos cervicais e amplitude de movimento da coluna cervical em indivíduos com e sem DTM
}

\author{
Electrical activity of cervical muscles and range of movement of cervical column in \\ individuals with and without DTM
}

Jovana de Moura Milanesi ${ }^{1}$, Eliane Castilhos Rodrigues Corrêa ${ }^{2}$, Graciele Silva Borin ${ }^{1}$, Juliana Alves Souza ${ }^{3}$, Fernanda Pasinato ${ }^{3}$

Estudo desenvolvido no Laboratório de Motricidade Orofacial do Programa de Pós-Graduação em Distúrbios da Comunicação Humana da Universidade Federal de Santa Maria (UFSM) - Santa Maria (RS), Brasil.

${ }^{1}$ Fisioterapeuta; Mestranda do Programa de Pós-Graduação em Distúrbios da Comunicação Humana pela UFSM - Santa Maria (RS), Brasil.

${ }^{2}$ Fisioterapeuta; Prof ${ }^{a}$ Adjunta do Departamento de Fisioterapia e Reabilitação e do Programa de Pós-Graduação em Distúrbios da Comunicação Humana da UFSM Santa Maria (RS), Brasil.

${ }^{3}$ Fisioterapeuta; Mestre em Distúrbios da Comunicação Humana pela UFSM - Santa Maria (RS), Brasil.

\section{ENDEREÇO PARA CORRESPONDÊNCIA}

Jovana de Moura Milanesi -

Alameda Santiago do Chile, 171/201 - Nossa Senhora das Dores - CEP: 97050-685 - Santa Maria (RS), Brasil-E-mail: jovanamil@ yahoo.com.br

APRESENTAÇÃO ago. 2010

ACEITO PARA PUBLICAÇÃO maio 2011

FONTE DE FINANCIAMENTO nenhuma

CONFLITO DE INTERESSE nada a declarar

\section{APRESENTAÇÃO EM EVENTO CIENTÍFICO}

Resumo apresentado no XVIII Congress of the International Society of Electrophysiology and Kinesiology, em Aalborg, Dinamarca

Aprovação do Comitê de Ética e Pesquisa da UFSM com parecer favorável número 0048.0.243.000-08
RESUMO: O objetivo da pesquisa foi avaliar a atividade elétrica e a dor à palpação dos músculos cervicais, a amplitude de movimento (ADM) da coluna cervical e a relação entre atividade elétrica e ADM cervical em indivíduos com e sem desordem temporomandibular (DTM). Participaram do estudo 53 voluntários, sendo 24 com DTM e 29 sem, de ambos os gêneros, com 18 a 32 anos de idade. A DTM foi diagnosticada pelo critério de diagnóstico em pesquisa para DTM (Research Diagnostic Criteria for Temporomandibular Disorders). A eletromiografia dos músculos esternocleidomastoídeo (ECOM) e trapézio superior foi realizada bilateralmente, na situação de repouso, com frequência de $2 \mathrm{KHz}$ e filtro passa-faixa de $10-1000 \mathrm{~Hz}$. As medidas de ADM de flexão, extensão, lateroflexão e rotação foram realizadas com um flexímetro e a comparação entre os grupos contou com o teste de Mann Whitney. O teste de Spearman foi aplicado para a correlação entre as variáveis, com nível de significância de $5 \%$. Não foi verificada nenhuma diferença na ADM cervical entre os grupos. A atividade elétrica demonstrou-se significativamente maior nos músculos ECOM direito $(p=0,0130)$, trapézio superior direito $(p=0,0334)$ e esquerdo $(p=0,0335)$ no grupo DTM em relação ao grupo controle. Não houve correlação significante entre atividade elétrica e ADM cervical. A dor nos músculos cervicais apresentou-se significativamente maior apenas no músculo ECOM direito $(p=0,0055)$. Conclui-se que os grupos estudados registraram diferença na atividade elétrica dos músculos cervicais, sendo esta maior em indivíduos com DTM.

Descritores: transtornos da articulação temporomandibular; eletromiografia; amplitude de movimento articular.

ABSTRACT: The aim of the research was to evaluate the electrical activity and pain on palpation of the cervical muscles, the cervical range of motion (ROM) and the relationship between electrical activity and ROM in individuals with and without temporomandibular disorder (TMD). Fifty three volunteers took part in the study, being 24 with TMD and 29 without, both genders, from 18 to 32 years old. The TMD was diagnosed through Research Diagnostic Criteria for Temporomandibular Disorder (RDC/TMD). The electromyografhy of Sternocleidomastoideus (SCM) and upper trapezius muscles was carried out bilaterally during rest situation, with a frequency of $2 \mathrm{KHz}$ and band-pass filter of $10-1000 \mathrm{~Hz}$. The measures of flexion, extension, lateral tilt and rotation ROM were acquired through a fleximeter and the comparison between groups was analyzed by Mann-Whitney test. The Spearman test verified the correlation between the variables, with $5 \%$ of significance level. There were no differences in the cervical ROM between the groups. The electrical activity was significantly higher in the right SCM $(p=0,0130)$, left $(p=0,0335)$ and right $(p=0,0334)$ trapezius muscles in the TMD group. There were no significant correlations between electrical activity and cervical ROM. Pain in the neck muscles was significantly higher only in the right SCM $(p=0,0055)$. It is concluded that the studied groups had difference in the electrical activity of the cervical muscles, which was larger in the individuals with TMD.

KeYwords: temporomandibular joint disorders; electromyography; range of motion, articular. 


\section{INTRODUÇূ̃̃}

A desordem temporomandibular (DTM) inclui distúrbios relacionados à articulação temporomandibular e ao complexo muscular mastigatório/cervical, sendo sua etiologia multifatorial ${ }^{1}$. Para o correto diagnóstico e manejo da DTM, a eletromiografia de superfície é um método quantitativo e não-invasivo muito utilizado ${ }^{2,3}$.

Eriksson et al. ${ }^{4}$ sugerem que movimentos funcionais mandibulares são resultado de uma ação coordenada dos músculos mandibulares e cervicais que levam a movimentos simultâneos na articulação temporomandibular, atlanto-occiptal e outras articulações da coluna cervical. Considerando esta ligação anatômica, Wiesinger et al. ${ }^{5}$ mostram a existência de uma forte comorbidade entre DTM e cervicalgia, sugerindo que podem compartilhar fatores de risco ou até influenciar uma a outra. O músculo esternocleidomastóideo (ECOM), especialmente, parece sofrer influência durante funções estomatognáticas devido seu papel estabilizador da cabeça $a^{6,7}$. Stiesch-Scholz et al. ${ }^{8}$ recomendam a realização de uma avaliação específica da coluna cervical e região da cintura escapular, com palpação muscular e testes de amplitude de movimento (ADM) passiva e ativa, uma vez que a disfunção silenciosa da região cervical pode ser esperada em indivíduos portadores de DTM principalmente com componente miogênico.

Considerando a relação da DTM com a coluna cervical e o fato de não se conhecer nenhum estudo que aborde as variáveis ADM cervical e eletromiografia dos músculos cervicais em pacientes com DTM, torna-se relevante a realização desta pesquisa que teve como objetivo avaliar a atividade elétrica e a dor à palpação dos músculos cervicais, a amplitude de movimento da coluna cervical e a relação entre atividade elétrica e ADM cervical em indivíduos com e sem desordem temporomandibular.

\section{METODOLOGIA}

O presente estudo foi aprovado pelo Comitê de Ética em Pesquisa da Universidade Federal Santa Maria (UFSM) e todos os voluntários assinaram o Termo de Consentimento Livre e Esclarecido (TCLE).

Estabeleceram-se como critérios de inclusão a presença de DTM para o grupo de estudo (GE) e a ausência de DTM para o grupo de controle (GC). Para ambos, os participantes deveriam ser dos dois gêneros e ter entre 18 e 32 anos de idade. Como critérios de exclusão consideraramse comprometimento neuropsicomotor, doença musculoesquelética, traumas ortopédicos, cirurgia prévia e/ou má formação na região craniofacial. Os participantes não poderiam estar ou terem sido submetidos a tratamento fisioterapêutico nos últimos seis meses.

Os 53 voluntários foram distribuídos, conforme a presença ou ausência de DTM verificada pelo critério de diagnóstico em pesquisa para DTM (RDC/TMD) ${ }^{9}$, em dois grupos: GE, composto por 24 sujeitos com DTM, sendo 3 homens e 21 mulheres, com idade média de 25,4₫4,2 anos; e o GC, com 29 indivíduos, 2 homens e 27 mulheres, com 22,3 $\pm 2,1$ anos.

Os voluntários foram submetidos ao registro eletromiográfico dos músculos cervicais (esternocleidomastóideo e fibras superiores do trapézio) na postura ortostática relaxada, sem se movimentarem durante 20 segundos. Previamente à coleta do sinal Eletromiográfico (EMG), foi realizada a limpeza da pele com uso de algodão embebido em álcool etílico $70^{\circ}$ para reduzir a sua impedância. Eletrodos de $\mathrm{Ag} / \mathrm{AgCl}$ do tipo DOUBLE (Hal Indústria e Comércio Ltda.) com formato circular, distância inter-eletrodo fixa de 20 mm, diâmetro de 10 mm, 2 mm de superfície de contato, ganho de 20 vezes, impedância de entrada de $10 \mathrm{G} \Omega$ e taxa de rejeição de modo comum >100 dB foram ligados a pré-amplificadores ativos com entrada diferencial (PA1020) da Lynx Tecnologia Eletrônica Ltda. Foram realizados testes de função muscular para correta colocação dos eletrodos. Para o teste do músculo ECOM, pediu-se ao voluntário posicionar e manter a cabeça para frente enquanto o examinador aplicava resistência manual sobre a região da glabela. Para o teste do músculo trapézio superior, o examinador aplicava resistência manual sobre os ombros do voluntário enquanto este realizava o movimento de elevação dos ombros contra a mesma ${ }^{10}$. Os eletrodos foram posicionados sobre a região do músculo ECOM anteriormente ao processo mastóide a uma distância de um terço entre este e o esterno, e no músculo trapézio (fibras superiores) os eletrodos foram posicionados na região média entre a coluna vertebral no nível da sétima vértebra cervical e o acrômio conforme Cram, Kasman \& Holtz ${ }^{11}$. Um eletrodo de referência foi colocado no esterno $^{11}$.

O exame eletromiográfico foi realizado com o um eletromiógrafo Lynx (Lynx Tecnologia Eletrônica Ltda.) de 8 canais com placa de conversão analógico-digital de 16 bits modelo CAD 10/26, cujos sinais foram condicionados por meio de amplificadores conforme as normas internacionais $^{12}$ com frequência de amostragem de 2 khz, filtros com frequência de corte passa alta de $10 \mathrm{~Hz}$ e passa baixa de $1000 \mathrm{~Hz}$. Devido aos diversos fatores que influenciam o sinal EMG e sua grande variabilidade, foram realizadas três coletas e, destas, selecionou-se aquela com menor ruído de interferência e melhor histograma ${ }^{13}$. O sinal EMG foi processado no domínio da amplitude, com medidas em root mean square (RMS) pelo software AqDAnalisys 7.0 (Lynx Electronic Technology Ltda.) e expressos em microvolts.

A ADM de flexão, extensão, inclinação direita e esquerda da coluna cervical foi aferida com o sujeito na posição sentada. As rotações direita e esquerda foram realizadas com o sujeito na posição supina $^{14}$. As posições para cada movimento avaliado, bem como a observação de possíveis padrões compensatórios nos movimentos, seguiram as recomendações do manual de utilização do aparelho. O flexímetro da marca Sanny ${ }^{\circledR}$ foi posicionado com velcro ao redor da cabeça, o mostrador posicionado na face lateral da cabeça para os movimentos de flexoextensão, na região frontal da cabeça para os movimentos de inclinação lateral direita e esquerda e no topo para os de rotação. Para a realização dos movimentos, os voluntários receberam as seguintes instruções: a) tentar encostar o queixo no peito para realizar a flexão; b) olhar para o teto para realizar a extensão; c) encostar a orelha no ombro direito e esquerdo para os movimentos de lateroflexão; d) olhar para lado direito e esquerdo para os movimentos de rotação. Foram realizadas três medidas (em graus) considerando-se a média das mesmas (Figura 1). 
A dor nos músculos cervicais foi verificada com palpação dos músculos trapézio superior e esternocleidomastoideo bilateralmente e realizada por um examinador devidamente treinado. A palpação seguiu a orientação do exame de banda tensa preconizado por Simons et al. ${ }^{15}$. O indivíduo foi orientado a permanecer na posição sentada com os braços apoiados sobre as pernas. $\mathrm{O}$ examinador utilizou a pegada em pinça com pressão de aproximadamente 1 Kgf, solicitando ao indivíduo informações sobre a presença de dor ou não conforme a graduação: sem dor, dor leve, dor moderada e dor grave. A classificação da gravidade da dor nos músculos cervicais foi adaptada do instrumento RDC/TMD, com base na palpação dos músculos mastigatórios ${ }^{9}$.

Tanto a avaliação da mobilidade cervical quanto a da dor à palpação muscular são parâmetros estabelecidos para detectar disfunção cervical (IDCC) conforme Wallace \& Klineberg ${ }^{16} \mathrm{e}$ tratam-se de métodos com confiabilidade já demonstrada ${ }^{14,17-19}$.

Os examinadores que realizaram a palpação muscular não conheciam os resultados do instrumento RDC quanto ao diagnóstico de DTM, sendo cegos em relação ao grupo que pertencia o participante. Examinadores diferentes realizaram a EMG e a fleximetria, portanto, não era conhecido pelo examinador o resultado das demais avaliações.

Para a análise estatística foi utilizado o programa STATISTICA 7 (Statistica for Windows - release 7.0 Stat Soft). A normalidade dos dados foi testada pelo teste Lilliefors. Como todos os dados não apresentaram distribuição normal, a comparação entre os grupos foi realizada pelo teste não-paramétrico de $U$ de Mann-Whitney. A correlação entre as variáveis ADM cervical e atividade elétrica muscular foi testada pelo teste Spearman, considerando $r>0,8$ para nível de correlação excelente; entre 0,6 e 0,8 para correlação moderada; de 0,3 a 0,5 para correlação fraca; e $r<0,3$ para correlação pobre ${ }^{20}$. Para análise estatística da dor à palpação, foram agrupadas as variáveis sem dor/dor leve e dor moderada/dor grave e aplicado o Teste do $\chi^{2}$. Aceitou-se nível de significância de 5\%.
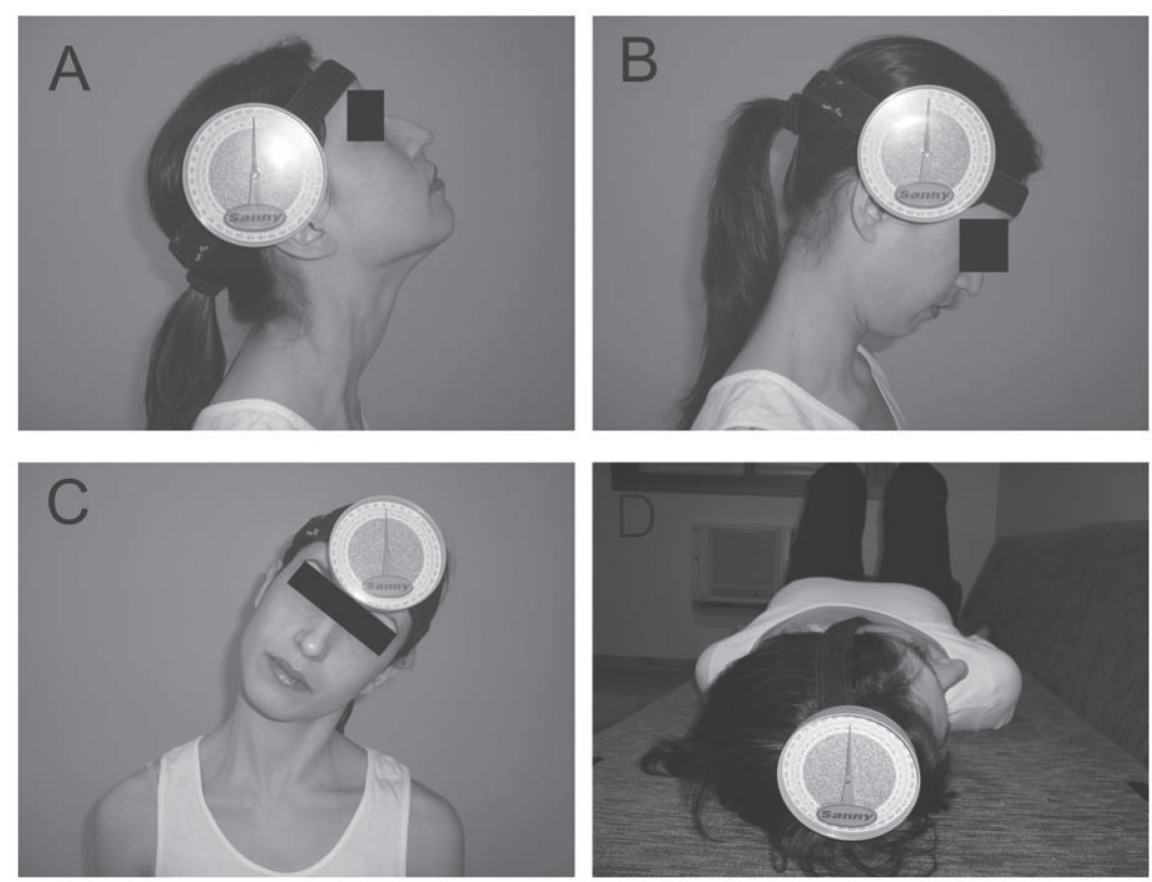

Figura 1. A: Movimento de extensão; B: Movimento de flexão; C: Movimento de lateroflexão; D: Movimento de rotação

Tabela 1. Média e Desvio Padrão (DP) dos valores de Root Mean Square $(\mu \mathrm{V})$ da atividade elétrica de repouso dos músculos cervicais nos grupos Desordem Temporomandibular (DTM) e Controle

\begin{tabular}{llll}
\hline \multirow{2}{*}{ Músculos } & \multicolumn{2}{c}{ Grupo DTM } & \multicolumn{2}{c}{ Grupo Controle } & \multirow{2}{*}{ Valor p } \\
\cline { 2 - 3 } & Média \pm DP & Média \pm DP & \\
\hline Esternocleidomastoídeo D & $5,15 \pm 1,35$ & $4,26 \pm 1,01$ & $0,0130^{*}$ \\
Esternocleidomastoídeo E & $4,87 \pm 1,58$ & $4,59 \pm 1,35$ & 0,6295 \\
Trapézio D & $11,22 \pm 11,62$ & $6,06 \pm 4,68$ & $0,0334^{*}$ \\
Trapézio E & $9,88 \pm 9,60$ & $7,25 \pm 5,46$ & $0,0335^{*}$ \\
\hline
\end{tabular}

*Teste $u$ de Mann-Whitney

RESULTADOS

Os valores da atividade eletromiográfica dos músculos cervicais no repouso foram maiores no grupo DTM do que no GC, com diferença estatisticamente significante nos músculos ECOM direito e trapézio superior direito e esquerdo (Tabela 1).

Os valores médios da ADM cervical do grupo DTM e controle encontram-se na Tabela 2. Não houve diferença significativa entre os grupos nos movimentos cervicais, porém o de extensão apresentou-se com amplitude levemente menor no DTM. Neste mesmo grupo foi verificada correlação negativa fraca entre a atividade elétrica do músculo ECOM direito e o movimento de extensão $(r=-0,33)$, músculos ECOM esquerdo e trapézio direito e o movimento de rotação à esquerda $(r=-0,37 ; r=-0,30)$ e entre o músculo trapézio direito e a inclinação à esquerda $(r=-0,42)$. Já no controle, observou-se correlação positiva fraca entre a atividade elétrica do músculo ECOM direito e o movimento de inclinação à esquerda $(r=0,34)$, trapézio direito e rotação à esquerda $(r=0,33)$ bem como correlação negativa fraca entre o músculo trapézio direito e o movimento de inclinação à direita $(r=-0,34)$. As demais correlações entre atividade elétrica de repouso dos músculos cervicais e a ADM cervical mostraram-se pobres.

A presença de dor nos músculos cervicais, nos grupos DTM e controle está expressa na Tabela 3, com diferença significativa no músculo ECOM direito. 
Tabela 2. Amplitude de movimento cervical (graus) nos grupos Desordem Temporomandibular (DTM) e Controle

\begin{tabular}{lcccc}
\hline $\begin{array}{l}\text { Amplitude de } \\
\text { movimento }\end{array}$ & Grupo DTM & Grupo Controle & & $\begin{array}{c}\text { Valores de } \\
\text { Média }\end{array}$ \\
\cline { 2 - 3 } normalidade de \\
Extensão & $71,88 \pm 12,70$ & $75,31 \pm 7,90$ & 0,3467 & $48-69^{\circ}$ \\
Flexão & $62,92 \pm 13,57$ & $57,52 \pm 8,95$ & 0,1758 & $61-93^{\circ}$ \\
Inclinação D & $45,69 \pm 7,53$ & $43,83 \pm 5,05$ & 0,3515 & $38-49^{\circ}$ \\
Inclinação E & $46,69 \pm 7,03$ & $45,69 \pm 5,56$ & 0,5954 & \\
Rotação D & $76,06 \pm 7,81$ & $73,41 \pm 7,43$ & 0,2046 & $70-92^{\circ}$ \\
Rotação E & $76,46 \pm 10,77$ & $76,17 \pm 7,95$ & 0,9928 & \\
\hline
\end{tabular}

*Chen et al.

DP: Desvio padrão; ADM: Amplitude de movimento

Tabela 3. Dor à palpação nos músculos cervicais nos grupos Desordem Temporomandibular (DTM) e Controle

\begin{tabular}{lrrrrr}
\hline \multirow{2}{*}{ Variáveis } & \multicolumn{2}{c}{ Grupo DTM } & \multicolumn{2}{c}{ Grupo Controle } & \multirow{2}{*}{ Valor $p$} \\
\cline { 2 - 5 } & $\mathrm{n}$ & $\%$ & $\mathrm{n}$ & $\%$ & \\
\hline Trapézio D & 5 & 20,87 & 8 & 27,58 & 0,5659 \\
$\quad$ Sem dor/dor leve & 19 & 79,20 & 21 & 72,42 & \\
$\quad$ Dor moderada/dor grave & & & & & \\
$\quad$ Trapézio E & 3 & 12,50 & 7 & 24,14 & 0,2811 \\
$\quad$ Sem dor/dor leve & 21 & 87,50 & 22 & 75,86 & \\
$\quad$ Dor moderada/dor grave & & & & & \\
Ecom D & 3 & 12,50 & 14 & 48,27 & $0,0055^{*}$ \\
$\quad$ Sem dor/dor leve & 21 & 87,50 & 15 & 51,73 & \\
$\quad$ Dor moderada/dor grave & & & & & \\
$\quad$ Ecom E & 3 & 12,50 & 7 & 37,93 & 0,1784 \\
$\quad$ Sem dor/dor leve & 21 & 87,50 & 18 & 62,07 & \\
$\quad$ Dor moderada/dor grave & & & & & \\
\hline
\end{tabular}

Ecom: Esternocleidomastoídeo

*Teste do $\chi^{2}$

DISCUSSÃO

Existem evidências anatômicas que comprovam a interação entre movimentos mandibulares com articulações e músculos cervicais ${ }^{4,21}$, o que justifica o estudo da ADM cervical na DTM. Embora não se conheçam trabalhos que estudaram conjuntamente a eletromiografia dos músculos cervicais e a medida da amplitude do movimento cervical em indivíduos com DTM, uma relação entre estes instrumentos foi parcialmente estabelecida por Aguilera et al. ${ }^{22}$. Estes pesquisadores utilizaram diferentes tratamentos miofasciais para pontos gatilhos latentes do músculo confiabilidade e aplicabilidade confirmadas para medidas de movimentos cervicais, inclusive em crianças ${ }^{14,17-19}$.

Os resultados desta pesquisa mostram associação entre a DTM e a disfunção cervical, uma vez que o grupo com DTM apresentou maior atividade elétrica de repouso dos músculos cervicais quando comparado ao GC. Também considerando esta relação, Wiesinger et al. ${ }^{5}$ avaliaram 616 pacientes quanto a sintomas de dor cervical e DTM, concluindo haver forte comorbidade e influências mútuas entre as duas situações. Ainda, Bevilaqua-Grossi et al. ${ }^{23}$, ao avaliar ambas as patologias, sugerem que os sinais e sintomas cervicais acompanham a gravidade da DTM, mas sinais e sintomas de DTM não aumentam com gravidade da disfunção cervical.

Maior atividade elétrica no repouso dos músculos ECOM direito e trapézio superior direito e esquerdo foi encontrada no grupo DTM. A presença de hiperatividade é considerada quando a elétrica muscular apresenta-se com valor superior a 5 microvolts ${ }^{11}$. Neste estudo, os músculos trapézio superior direito e esquerdo mostraram-se hiperativos, o que pode ser atribuído ao fato de que o músculo trapézio é sistematicamente recrutado para produzir estabilidade do pescoço e pode ser ativado por reflexos nociceptivos presentes em mecanismos protetores ${ }^{24}$. Ao avaliar 38 indivíduos com DTM miogênica, Pallegama et al. ${ }^{25}$ verificaram aumento na atividade elétrica de repouso relacionada à presença de dor nos músculos ECOM e trapézio nesses indivíduos quando comparados a controles. Ciunffolo et al. ${ }^{26}$ observaram coativação dos ECOMs durante a máxima intercuspidação. Tais achados sustentam a relação existente entre as regiões crânio-mandibular e cervical, na qual os movimentos funcionais mandibulares envolvem tanto a mandíbula quanto o complexo cabeça-pescoçO ${ }^{21,27}$.

Apesar de maior atividade elétrica dos músculos cervicais nos indivíduos com DTM, não foi encontrada nenhuma diferença estatisticamente significante na amplitude de movimento cervical entre os grupos. A ADM cervical depende de inúmeras variáveis, como idade, gênero, dimensão do pescoço e dor ${ }^{28-31}$. Reynolds et al. ${ }^{28}$ avaliaram a ADM cervical e a dimensão do pescoço em 100 sujeitos saudáveis com idades entre 20 e 40 anos. 
Seus achados mostraram que o movimento de flexo-extensão está relacionado à circunferência do pescoço e o movimento de inclinação lateral com a razão entre a circunferência e comprimento do pescoço. As variáveis referentes à dimensão do pescoço não foram avaliadas neste estudo.

A influência da idade no movimento cervical foi constatada por um estudo de Lansade et al. ${ }^{29}$ com 140 indivíduos assintomáticos de ambos os sexos e idade entre 20 e 93 anos. Os autores concluíram que a ADM cervical decresce aproximadamente $5,5^{\circ}$ a $7,9^{\circ}$ por década, mais significantemente a partir dos 60 anos. Contrariamente, Smith et al. ${ }^{30}$ não encontraram diferenças na ADM cervical em uma amostra de 66 indivíduos com idade média de 33 anos $( \pm 13,5)$, mas sugerem que o gênero e a presença de dor devem ser considerados fatores contribuintes para a limitação do movimento cervical.

O fato de esta pesquisa não ter encontrado diferenças na ADM cervical entre os grupos pode ser justificado pela baixa faixa etária dos sujeitos avaliados (média de 25,4 anos $\pm 4,18$ ) e a adaptabilidade do sistema músculo esquelético à patologia. Ries e Bérzin ${ }^{10}$ afirmam que compensações podem representar um mecanismo necessário para fornecer estabilidade para os sistemas mandibular e cervical, sendo necessário incluir a avaliação clínica da coluna cervical em indivíduos com DTM. Ainda, tanto os indivíduos com DTM quanto o GC apresentaram valores de ADM cervical semelhantes ao considerado normal estabelecido em uma meta-análise por Chen et al. ${ }^{32}: 48^{\circ}$ a $69^{\circ}$ para flexão; $61^{\circ}$ a $93^{\circ}$ para extensão; $38^{\circ}$ a $49^{\circ}$ para as inclinações laterais direita ou esquerda; e $70^{\circ}$ a $92^{\circ}$ para as rotações direita ou esquerda.

Sabe-se que a dor cervical é um fator que interfere nos movimentos de cabeça e pescoço ${ }^{31,33}$. Ferrão \& Traebert $^{34}$ afirmam que pontos de tensão nas estruturas cervicais levam ao aumento da dor, que pode impedir a completa ADM. Entretanto, na presente pesquisa, ambos os grupos apresentaram alguma dor à palpação e somente o ECOM direito foi mais sensível nos indivíduos com DTM, sem diferença entre os grupos na ADM cervical. Neste caso, a dor parece não ter sido um fator limitante para a ADM cervical dos grupos avaliados. Lee et al. ${ }^{31}$ avaliaram a ADM cervical em indivíduos com e sem dor subclínica, porém com idade entre 22 e 42 anos, e encontraram prejuízos nos movimentos de rotações e extensão no grupo com dor quando comparado ao controle. Ainda, Stiesch-Scholz et al. ${ }^{8}$, ao investigar a coluna cervical e cintura escapular de indivíduos com DTM, encontraram correlação positiva entre DTM e disfunção cervical com maior sensibilidade nos músculos cervicais destes quando comparados a controles. Os autores afirmam que esta correlação pode ser explicada pela estreita ligação funcional entre os sistemas mastigatório e craniocervical. Apesar da relação, o critério de diagnóstico em pesquisa para DTM (RDC/TMD) desenvolvido por Dworkin e Le Resche ${ }^{9}$ não contempla a avaliação da coluna cervical, podendo constituir limitação no diagnóstico mais abrangente da DTM.

Por fim, as correlações entre a atividade elétrica muscular de repouso e a ADM cervical apresentaram-se fracas. O fato de não se verificar esta correlação pode ser devido às ADMs dentro da normalidade em ambos os grupos. Ainda, apesar da maior atividade elétrica dos músculos cervicais nos indivíduos com DTM, não se pode afirmar que este seja um fator de limitação do movimento cervical.

\section{CONCLUSÃO}

Com base nos resultados desta pesquisa, pode-se concluir que os grupos estudados apresentaram diferença na atividade elétrica dos músculos cervicais, sendo esta mais elevada em indivíduos com DTM. Os grupos foram semelhantes quanto à ADM da coluna cervical, apresentando-se em níveis normais. Com isso, verificaram-se correlações fracas entre a ADM cervical e a atividade elétrica dos músculos cervicais. A dor nos músculos cervicais foi significativamente maior apenas no músculo ECOM direito no grupo DTM e não influenciou a sua atividade EMG, assim como a ADM cervical. Sugere-se a realização de estudos com amostra de faixa etária mais elevada e avaliação de variáveis como a gravidade da DTM.

\section{REFERÊNCIAS}

1. Biasotto-Gonzalez DA. Abordagem interdisciplinar das disfunções temporomandibulares. São Paulo: Manole; 2005.

2. Landulpho AB, Silva WA, Silva FA, Vitti M.

Electromyographic evaluation of masseter and anterior temporalis muscles in patients with temporomandibular disorders following interocclusal appliance treatment. J Oral Rehabil. 2004;31(2):95-8.

3. Ferrario VF, Tartaglia GM, Luraghi FE, Sforza C. The use of surface electromyography as a tool in differentiating temporomandibular disorders from neck disorders. Man Ther. 2007;12(4):372-9.

4. Eriksson PO, Häggman-Henrikson B, Nordh E, Zafar H. Co-ordinated mandibular and head-neck movements during rhythmic jaw activities in man. J Dent Res. 2000;79(6):1378-84.

5. Wiesinger B, Malker H, Englund E, Wänman A. Does a dose-response relation exist between spinal pain and temporomandibular disorders? BMC Musculoskelet Disord. 2009;10:28.

6. Davies PL. Electromyographic study of superficial neck muscles in mandibular function. J Dent Res. 1979;58(1):537-8.

7. Chandu A, Suvinen TI, Reade PC, Borromeo GL. Electromyographic activity of frontalis and sternocleidomastoid muscles in patients with temporomandibular disorders. J Oral Rehabil. 2005;32(8):571-6. 


\section{Referências (cont.)}

8. Stiesch-Scholz M, Fink M, Tschernitschek H. Comorbidity of internal derangement of the temporomandibular joint and silent dysfunction of the cervical spine. J Oral Rehabil. 2003;30(4):386-91.

9. Dworkin SF, LeResche L. Research diagnostic criteria for temporomandibular disorders: review, criteria, examinations and specifications, critique. J Craniomandib Disord. 1992;6(4):301-55.

10. Ries LGK, Bérzin F. Cervical pain in individuals with and without temporomandibular disorders. Braz J Oral Sci. 2007;6(20):1301-7.

11. Cram JR; Kasman GS; Holtz J. Introduction to surface electromyography. Gaithersburg, Maryland: Aspen; 1998.

12. Merletti R. Standards for Reporting EMG Data. Boston; 2004 [citado 30 set 2004] Disponível em: http://www. isek-online.org/standards_emg.html

13. Ferla A, Silva AM, Côrrea EC. Electrical activity of the anterior temporal and masseter muscles in mouth and nasal breathing children. Braz J Otorhinolaryngol. 2008;74(4):588-95.

14. Chaves TC, Nagamine HM, Belli JFC, Hannai MCT, Bevilaqua-Grossi D, Oliveira AS. Confiabilidade da fleximetria e goniometria na avaliação da amplitude de movimento cervical em crianças. Rev Bras Fisioter. 2008;12(4):283-9.

15. Simons DG, Travell JG, Simons LS. Dor e disfunção miofascial: manual dos pontos gatilho. $2^{\mathrm{a}}$ ed. Porto Alegre: Artmed; 2005.

16. Wallace C, Klineberg IJ. Management of craniomandibular disorders. Part 1: A craniocervical dysfunction index. J Orofac Pain. 1993;7(1):83-8.

17. Piva SR, Erhard RE, Childs JD, Browder DA. Inter-tester reliability of passive intervertebral and active movements of the cervical spine. Man Ther. 2006;11(4):321-30.

18. Prushansky T, Dvir Z. Cervical motion testing: methodology and clinical implications. J Manipulative Physiol Ther. 2008;31(7):503-8.

19. Williams MA, McCarthy CJ, Chorti A, Cooke MW, Gates S. A systematic review of reliability and validity studies of methods for measuring active and passive cervical range of motion. J Manipulative Physiol Ther. 2010;33(2):138-55.

20. Chan YH. Bioestatistics 104: correlational analyis. Singapore Med J. 2003;44(12):614-9.

21. Cuccia A, Caradonna C. The relationship between the stomatognathic system and body posture. Clinics. 2009;64(1):61-6.
22. Aguilera FJ, Martín DP, Masanet RA, Botella AC, Soler LB, Morell FB. Immediate effect of ultrasound and ischemic compression techniques for the treatment of trapezius latent myofascial trigger points in healthy subjects: a randomized controlled study. J Manipulative Physiol Ther. 2009;32(7):515-20.

23. Bevilaqua-Grossi D, Chaves TC, Oliveira AS. Cervical spine signs and symptoms: perpetuating rather than predisposing factors for temporomandibular disorders in women. J Appl Oral Sci. 2007;15(4):259-64.

24. Ritzel CH, Diefenthaeler F, Rodrigues AM, Guimarães ACS, Vaz MA. Temporomandibular joint dysfunction and trapezius muscle fatigability. Rev Bras Fisioter. 2007;11(5):333-9.

25. Pallegama RW, Ranasinghe AW, Weerasinghe VS, Sitheeque MA. Influence of masticatory muscle pain on electromyographic activities of cervical muscles in patients with myogenous temporomandibular disorders. J Oral Rehabil. 2004;31(5):423-9.

26. Ciuffolo F, Manzoli L, Ferritto AL, Tecco S, D'Attilio M, Festa F. Surface electromyographic response of neck muscles to maximal voluntary clenching of the teeth. J Oral Rehabil. 2005;32(2):79-84.

27. Schindler HJ, Lenz J, Türp JC, Schweizerhof K, Rues S. Influence of neck rotation and neck lateroflexion on mandibular equilibrium. J Oral Rehabil. 2010;37(5):329-35.

28. Reynolds J, Marsh D, Koller H, Zenenr J, Bannister G. Cervical range of movement in relation to neck dimension. Eur Spine J. 2009;18(6):863-8.

29. Lansade C, Laporte S, Thoreux P, Rousseau MA, Skalli W, Lavaste F. Three-dimensional analysis of the cervical spine kinematics: effect of age and gender in healthy subjects. Spine. 2009;34(26):2900-6.

30. Smith K, Hall T, Robinson K. The influence of age, gender, lifestyle factors and sub-clinical neck pain on the cervical flexion-rotation test and cervical range of motion. Man Ther. 2008;13(6):552-9.

31. Lee H, Nicholson LL, Adams RD. Cervical range of motion associations with subclinical neck pain. Spine. 2004;29(1):33-40.

32. Chen J, Solinger AB, Poncet JF, Lantz CA. Meta-analylis of normative cervical motion. Spine. 1999;24(15):1571-8.

33. Vogt L, Segieth C, Banzer W, Himmelreich H. Movement behavior in patients with chronic neck pain. Physiother Res Int. 2007;12(4):206-12.

34. Ferrão MIP, Traebert J. Prevalence of temporomandibular disfunction in patients with cervical pain under physiotherapy treatment. Fisioter Mov. 2008;21(4):63-70. 